\title{
LEIOMIOMATOSIS PARÁSITA EN MEDELLÍN, COLOMBIA: REPORTE DE UN CASO Y REVISIÓN DE LA LITERATURA
}

\section{Parasitic leiomyomatosis in Medellín, Colombia: A case report and review of the literature} Gonzalo Ángel-Cano, $M D^{1}$; Javier Castro-Solís, $\mathrm{MD}^{2}$; Adriana Arango-Martínez, MD, $\mathrm{MSc}^{3}$

Recibido: mayo 21/13 - Aceptado: junio 12/14

\section{RESUMEN}

Objetivo: mostrar un caso de leiomiomatosis parásita que se presentó después de miomectomía laparoscópica y revisar la literatura disponible respecto a la histopatología, la fisiopatología, el diagnóstico y el tratamiento de la entidad.

Materiales y métodos: se presenta el caso de una paciente de 30 años con antecedente de miomectomía por laparoscopia, que consultó a la clínica las Americas (Medellín, Colombia), centro privado de atención, por sangrado uterino de un mes de evolución. Por ultrasonido pélvico se documentó la presencia de leiomiomas uterinos. Fue sometida a nueva laparoscopia que reveló cuatro miomas parásitos en la cavidad pélvica. Se realizó una búsqueda en las bases de datos Medline vía PubMed y SciELO con los términos MeSH: "leiomyomatosis parasitic", "myomectomy", "laparoscopic" y "myoma”, y los términos DeCS: "mioma”, "leiomiomatosis parásito" "miomectomía" y "laparoscopia".

Resultados: se seleccionaron 12 artículos de los cuales 10 son reporte de caso, una serie de casos y una revisión narrativa de la literatura.

1 Ginecólogo oncólogo, Instituto de Cancerología, Clínica Las Américas, Medellín, Colombia. jcastros1975@yahoo.com

2 Residente de Cirugía laparoscópica ginecológica, Universidad CES, Medellín, Colombia.

3 Ginecóloga laparoscopista, magíster en epidemiología, docente Universidad CES, Medellín, Colombia.
Conclusión: la leiomiomatosis parásita es un evento que se presenta en la actualidad más frecuentemente después de miomectomía laparoscópica y que puede llegar a requerir manejo quirúrgico.

Palabras clave: leiomiomatosis, mioma, laparoscopia, miomectomía, parásito.

\section{ABSTRACT}

Objective: To present a case of parasitic leiomyomatosis occurring after laparoscopic myomectomy, and a review of the literature on the histopathology, pathophysiology, diagnosis, and treatment of this condition.

Materials and methods: 30 year-old female patient with a history of laparoscopic myomectomy who presented to Clínica las Américas (Medellín, Colombia), a private healthcare centre, complaining of uterine bleeding lasting one month. The presence of leiomyomas was document on ultrasound. The patient was taken again to laparoscopy and four parasitic leiomyomas were found in the pelvic cavity. A search was conducted in the Medline databases through PubMed and SciELO using the MeSH terms "leiomyomatosis, parasitic", "myomectomy", "laparoscopic", "myoma", and the DeCS terms "myoma", "parasite leiomyomatosis", "myomectomy" and "laparoscopy". 
Results: Overall, 12 articles were selected, including 10 case reports, 1 case series and 1 narrative review of the literature.

Conclusion: Parasitic leiomyomatosis is an event found most frequently at the present time after laparoscopic myomectomy, and may require surgical management.

Key words: Leiomyomatosis, myoma, laparoscopy, myomectomy, parasite.

\section{INTRODUCCIÓN}

La leiomiomatosis parásita (LP) fue descrita por primera vez en 1909 por Kelly y Cullen como el hallazgo de miomas implantados en la superficie de otros órganos por separación espontánea del útero de miomas subserosos pediculados (1). Se conoce poco sobre la etiopatogenia e historia natural de esta enfermedad, y la literatura disponible se limita a reportes aislados o a series de casos (2). En la última década se ha incrementado el número de casos reportados de LP de forma paralela al creciente auge del manejo mínimamente invasivo para la miomatosis uterina $(3,4)$. Aunque su frecuencia es desconocida, un estudio retrospectivo informa una incidencia 1,2\% de las pacientes sometidas previamente a miomectomía (5). Por esto es importante que el médico ginecólogo conozca sobre esta emergente forma de presentación de leiomiomatosis. El objetivo del presente artículo es reportar un caso clínico y revisar la literatura disponible respecto a su histopatología, fisiopatología, presentación clínica, diagnóstico y opciones de manejo.

\section{REPORTE DE CASO}

Paciente de 30 años, grávida; número de partos, uno, que consultó a la Clínica de las Américas -institución privada de atención general de alta complejidad ubicada en Medellín, Colombia, que atiende población del aseguramiento contributivo- por sangrado intermenstrual abundante de un mes de evolución. Como antecedente se le había practicado en la misma institución una miomectomía laparoscópica hacía un año, en la cual se resecó un mioma cornual izquierdo de $15 \mathrm{~cm}$ de diámetro que comprometía ligamento ancho, trompa y ligamento redondo ipsilateral, el cual fue extraído por fragmentación con morcelador. La paciente evolucionó satisfactoriamente después del procedimiento inicial. El reporte de patología confirmó el diagnóstico de fibroma uterino. En la revisión posoperatoria se le insertó un dispositivo intrauterino liberador de levonorgestrel. Permaneció asintomática hasta un mes antes de la consulta. Al examen físico se encontró útero aumentado de tamaño, doloroso a la palpación, de contornos irregulares. La ecografía pélvica sugería múltiples miomas de 2 a $3 \mathrm{~cm}$ de diámetro con predominio fúndico, por lo que se decidió realizar miomectomía laparoscópica. Se encontraron cuatro lesiones sésiles en superficie serosa de colon sigmoide y receso pararrectal izquierdo, la mayor de ellas de 2,5 cm x $1 \mathrm{~cm}$, además de 3 miomas subserosos en fondo uterino de $2 \mathrm{~cm}$ (figura 1). Las lesiones se resecaron en su totalidad por laparoscopia, fraccionándolas para su extracción con ayuda del morcelador, y se enviaron a estudio histológico. El procedimiento fue ambulatorio, sin complicaciones. El estudio histológico mostró un patrón con haces de células musculares lisas y zonas de degeneración hialina compatible con leiomiomatosis. Actualmente la paciente se encuentra asintomática, y el seguimiento ecográfico pélvico de control cuatro meses después no mostró lesiones agregadas.

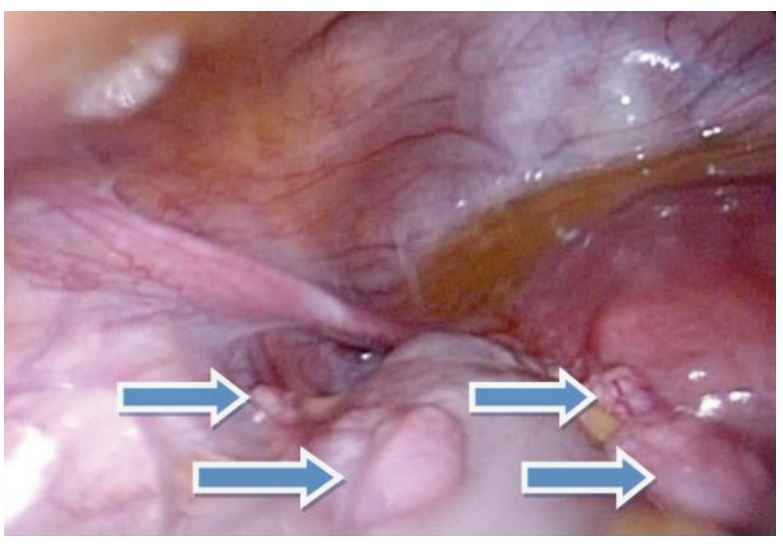

Figura 1. Leiomiomas parásitos sobre superficie de colon y pared pélvica 
Aspectos éticos. Se obtuvo autorización de la paciente para la publicación del presente artículo. Se salvaguardó la confidencialidad de su identidad.

\section{MATERIALES Y MÉTODOS}

Se realizó una búsqueda bibliográfica en las bases de datos Medline vía PubMed y SciELO con los términos MeSH: "leiomyomatosis parasitic", "myomectomy", "laparoscopic" y "myoma”, y los términos DeCS: "mioma", "leiomiomatosis parásito", "miomectomía” y "laparoscopia”. La búsqueda se limitó a los últimos veinticinco años.

\section{RESULTADOS}

Se seleccionaron 12 artículos de los cuales 10 son reporte de caso, una serie de casos y una revisión narrativa de la literatura

Anatomopatología. La LP es una entidad de comportamiento benigno que histológicamente se caracteriza por la presencia de nódulos de células musculares lisas y fibroblastos en la superficie peritoneal de la cavidad abdominal o pélvica. Estos nódulos pueden hallarse de manera incidental, en cirugías por otras indicaciones, o causar síntomas que hacen que la paciente consulte $(4,5)$. La mayoría de los implantes se han documentado en la superficie peritoneal parietal y visceral de la cavidad pélvica y en el epiplón; también se han descrito subdiafragmáticos y en las cicatrices de los puertos umbilicales o laterales; se han reportado masas de entre uno a treinta centímetros de diámetro (6-13).

Fisiopatología. Varios reportes de caso sugieren una relación entre miomectomía laparoscópica y leimomimatosis parásita $(7,10)$ (tabla 1). Esta asociación se explicaría en los recientes avances en cirugía laparoscópica, especialmente por el uso de morceladores eléctricos que fragmentan los miomas lo que permite resecar y extraer miomas uterinos que anteriormente solo se podían recuperar por colpotomía, o incisiones grandes en pared abdominal. Sin embargo, el proceso de fragmentación puede asociarse a la presencia de remanentes tisulares microscópicos que se implantan sobre el tejido peritoneal parietal o visceral de donde obtienen su propia vasculatura, bajo la influencia de factores hormonales e inmunológicos aún no bien esclarecidos $(3,5,7,10)$. Como soporte de esta teoría, en el año 2007 Takeda comparó las muestras de tejido obtenidas por resección de LP en una paciente, con fragmentos extraídos 6 años antes de la misma paciente durante una miomectomía laparoscópica encontrando idénticos patrones histológicos (10).

\begin{tabular}{|c|c|c|c|c|c|c|}
\hline \multicolumn{7}{|c|}{$\begin{array}{l}\text { Tabla } 1 . \\
\text { Series de casos reportados de leiomiomatosis parásita después de miomectomía } \\
\text { laparoscópica con uso de morcelador }\end{array}$} \\
\hline $\begin{array}{c}\text { Autor y año de } \\
\text { publicación }\end{array}$ & $\begin{array}{l}\text { Número de } \\
\text { pacientes }\end{array}$ & $\begin{array}{l}\text { Meses después } \\
\text { de primera } \\
\text { cirugía }\end{array}$ & Síntomas & $\begin{array}{l}\text { Número } \\
\text { de miomas } \\
\text { resecados }\end{array}$ & Localización & Manejo \\
\hline Sinha (2007) & 2 & 36 y 8 & $\begin{array}{l}\text { Masa } \\
\text { abdominal } \\
\text { y dolor }\end{array}$ & 4 & Pelvis & Laparoscopia \\
\hline Nezhat (2010) & 8 & 2 a 204 & $\begin{array}{l}\text { Dolor, } \\
\text { metrorragia, } \\
\text { sensación de masa }\end{array}$ & 15 & $\begin{array}{c}\text { Pelvis } 7 \\
\text { abdominal } 1\end{array}$ & Laparoscopia \\
\hline Larrain (2010) & 2 & 36 a 192 & Dolor pélvico & 2 & Pelvis & Laparoscopia \\
\hline Cuccinella (2011) & 4 & 24 a 108 & $\begin{array}{l}2 \text { asintomáticas } \\
2 \text { dolor pélvico } \\
\text { y masa palpable }\end{array}$ & 11 & $\begin{array}{l}\text { Pared abdominal } \\
\text { y pelvis }\end{array}$ & $\begin{array}{c}3 \\
\text { laparoscopia } \\
1 \text { laparotomía }\end{array}$ \\
\hline
\end{tabular}


Con base en esta posible asociación se ha sugerido que durante una miomectomía laparoscópica que implique la fragmentación de la pieza quirúrgica se deben hacer esfuerzos por extraer todos los pedazos de mioma, incluyendo una exhaustiva revisión de la cavidad abdominal y pélvica, con el objetivo de identificar y retirar cualquier porción de tejido que haya podido ser esparcida sobre las superficies peritoneales durante el proceso de morcelación (5).

En otras ocasiones los miomas parásitos se originan de miomas pediculados subserosos que pierden, por alguna razón, su sustento original y se siembran en la superficie peritoneal adyacente recibiendo nuevo aporte vascular $(1,3)$.

Presentación clínica, diagnóstico y manejo. Muchas veces esta entidad cursa de manera asintomática y, como se mencionó, solo se diagnostica incidentalmente como hallazgo quirúrgico; otras veces cursa con dolor abdominal, retención urinaria, sensación de masa, abdomen agudo por torsión, necrosis e incluso peritonitis $(6,7)$. En promedio, las pacientes son diagnosticadas después del segundo año de la cirugía inicial. El examen físico no es concluyente. Se ha informado que el ultrasonido y la resonancia mangética nuclear pueden ser útiles como ayuda diagnóstica, las imágenes típicas son nódulos sólidos de contornos regulares y tamaño variable dispersos en las superficies peritoneales $(3,5,7)$. El manejo en las pacientes sintomáticas es quirúrgico, por laparotomía o laparoscopia $(3,5)$.

\section{CONCLUSIONES}

La leiomiomatosis parásita es una patología que en ocasiones se presenta después de una miomectomía laparoscópica con morcelación; cuando se asocia a manifestaciones clínicas puede requerir manejo quirúrgico. Las pacientes que van a ser sometidas a miomectomía laparoscópica deben recibir información sobre esta patología. Se han descrito pocos casos, por lo que se necesitan estudios prospectivos que determinen la incidencia y aporten más información sobre la fisiopatología de esta enfermedad.

\section{REFERENCIAS}

1. Kelly HA, Cullen TS. Myomata of the uterus. Philadelphia (PA): WB Saunders; 1909. p. 13.

2. Sinha R, Sundaram M, Lakhotia S, Kadam P, Rao G, Majahan C. Parasitic myoma after morcellation. J Gynecol Endosc Surg. 2009;1:113-5.

3. Kho KA, Nezhat C. Parasitic Myomas. Obstet Gynecol. 2009;11:611-5.

4. Leren V, Langrebrekke A, Qvigstad E. Parasitic leiomyomas after laparoscopic surgery with morcellation. Acta Obstet Gynecol Scand. 2012; 91:1233-6.

5. Cuccinella G, Granese R, Calagna G, Somigliana E, Perino A. Parasitic myomas after laparoscopic surgery: an emergin complication in the use of morcellator? Report of four cases. Fertil Steril. 2011;96:90-6.

6. Dan D, Harnanan D, Hariharan S, Maharaj R, Hosein I, Naraynsingh V. Extrauterine leiomyomata presenting with sepsis requiring hemicolectomy. Rev Bras Ginecol Obstet. 2012;34:285-9.

7. Nezhat C, Kho K. Iatrogenic miomas: new class of myomas? J Minim Invasive Gynecol. 2010;17:545-0.

8. Epstein JH, Nejat EJ, Tsai T. Parasitic myomas after laparoscopic myomectomy: case report. Fertil Steril. 2009;91:13-4.

9. Paul PG, Koshy AK. Multiple peritoneal parasitic myomas after laparoscopic myomectomy and morcellation. Fertil Steril. 2006;85:492-3.

10. Takeda A, Mori M, Sakai K, Mitsui T, Nakamura H. Parasitic peritoneal leiomyomatosis diagnosed 6 years after laparoscopic myomectomy with electric tissue morcellation: report of a case and review of the literature. J Minim Invasive Gynecol. 2007;14:770-5.

11. Moon HS. Parasitic leiomyoma in the abdominal wall after laparoscopic myomectomy. Fertil Steril. 2008;90:1201-2.

12. Larrain D, Rabischong B, Khoo CK, Botchorishvili R, Canis M, Mage G. "Iatrogenic" parasitic myomas: unusual late complication of laparoscopic morcellation procedures. J Minim Invasive Gynecol. 2010;17:719-24.

13. Jebbunaher S, Begun SA. Parasitic leiomyoma: a case report. Mymensingh Med J. 2013;22:173-5. 